\title{
Electronic Impurity Doping in CdSe Nanocrystals
}

\author{
Ayaskanta Sahu, ${ }^{\dagger, \ddagger}$ Moon Sung Kang, ${ }^{\ddagger, \S}$ Alexander Kompch, ${ }^{\|}$Christian Notthoff, $/$Andrew W. Wills, ${ }^{\perp}$ \\ Donna Deng, " Markus Winterer," C. Daniel Frisbie, \\ ${ }^{\dagger}$ Optical Materials Engineering Laboratory, ETH Zurich, 8092 Zurich, Switzerland \\ ${ }^{\ddagger}$ Department of Chemical Engineering and Materials Science, University of Minnesota, Minneapolis, Minnesota 55455, United States \\ ${ }^{\S}$ Department of Chemical Engineering, Soongsil University, Seoul 156-743, Korea \\ "Nanoparticle Process Technology and Center for NanoIntegration Duisburg-Essen (CeNIDE), University Duisburg-Essen, D-47057 \\ Duisburg, Germany \\ ${ }^{\perp}$ Department of Chemistry, University of Minnesota, Minneapolis, Minnesota 55455, United States
}

\section{Supporting Information}

ABSTRACT: We dope CdSe nanocrystals with Ag impurities and investigate their optical and electrical properties. Doping leads not only to dramatic changes but surprising complexity. The addition of just a few $\mathrm{Ag}$ atoms per nanocrystal causes a large enhancement in the fluorescence, reaching efficiencies comparable to core-shell nanocrystals. While Ag was expected to be a substitutional acceptor, nonmonotonic trends in the fluorescence and Fermi level suggest that Ag changes from an interstitial ( $\mathrm{n}$ type) to a substitutional (p-type) impurity with increased doping.

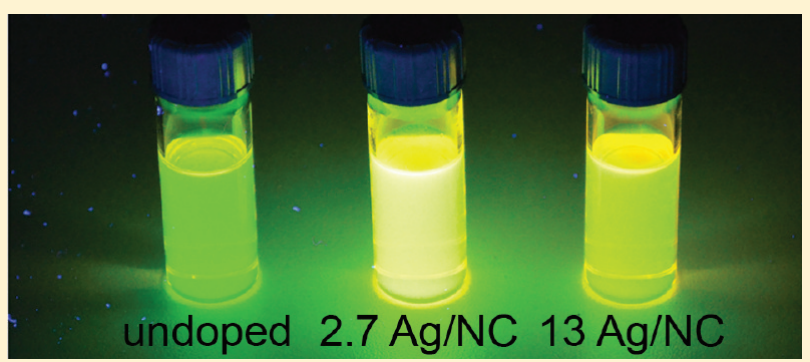

KEYWORDS: Semiconductor nanocrystals, colloidal quantum dots, silver doping, photoluminescence enhancement, cation exchange, thin-film transistors

$\mathrm{E}$ lectronic impurity doping is the process in which impurity atoms are intentionally added to bulk semiconductors to provide either extra electrons (n-type doping) or extra holes ( $\mathrm{p}$ type doping). The carriers then enable electrical transport through an otherwise poorly conducting material. Surprisingly, even after decades of research, little progress has been made on this process in colloidal quantum dots, also known as semiconductor nanocrystals. ${ }^{1,2}$ These materials exhibit sizedependent optical spectra, which can be useful in solar cells ${ }^{3}$ and solid-state lighting. ${ }^{4}$ For these applications, electrical current must be collected or injected from films of nanocrystals. Because the films are inherently insulating, the introduction of extra carriers is beneficial for enhancing conduction. For CdSe, the most studied nanocrystal system, several methods have provided the carriers, including placement of electron-donating molecules in the vicinity of the nanocrystal surface (remote doping $)^{5-7}$ and application of external electric fields (electrochemical doping). ${ }^{6-9}$ When carriers were added, conductivity was dramatically increased. ${ }^{6}$ In principle, it should also be possible to provide extra carriers to CdSe nanocrystals via impurity doping.

However, studies of electronic impurity doping in colloidal nanocrystals have been fairly limited. ${ }^{10-24}$ Development has been impeded by the broader synthetic challenge of incorporating an impurity into a nanoscale particle. ${ }^{25}$ While progress has been made with magnetic substitutional impurities in II-VI semiconductor nanocrystals, ${ }^{26}$ these dopants (e.g., Mn and $\mathrm{Co}$ ) are isovalent with the cations that they replace, and no additional carriers are introduced. Recently, efforts have attempted to incorporate heterovalent impurities that can provide these carriers, most notably in InAs. In Cd-doped InAs, conductivity through films was clearly affected, but the impurities were likely coating the nanocrystal surfaces. ${ }^{17}$ In $\mathrm{Cu}-, \mathrm{Ag}-$, and Au-doped samples, shifts in the valence and conduction bands were observed through scanning tunneling microscopy on individual nanocrystals, and a simple model assuming interstitial $\mathrm{Cu}$ and substitutional $\mathrm{Ag}$ and $\mathrm{Au}$ was proposed. ${ }^{19}$ However, both InAs studies ${ }^{17,19}$ focused on the heavily doped limit (tens to hundreds of dopants per nanocrystal). "Solotronic" behavior ${ }^{27}$ arising from a solitary or a few electronic dopants has not been fully explored in colloidal nanocrystals. Furthermore, little work has addressed electronic impurity doping in the prototypical system, CdSe. ${ }^{12,16,24}$

Here, we analyze CdSe nanocrystals that are lightly doped with Ag. Herein, we use the phrase "lightly doped" to indicate that only a few impurities are added to each nanocrystal. We note that in the language of bulk semiconductors, where the number of impurities is quoted per unit volume, the concentration of the dopants is still high. ${ }^{10}$ Of course, studying this limit where a small number of impurities are confined in a

Received: March 5, 2012

Revised: April 24, 2012

Published: April 25, 2012 

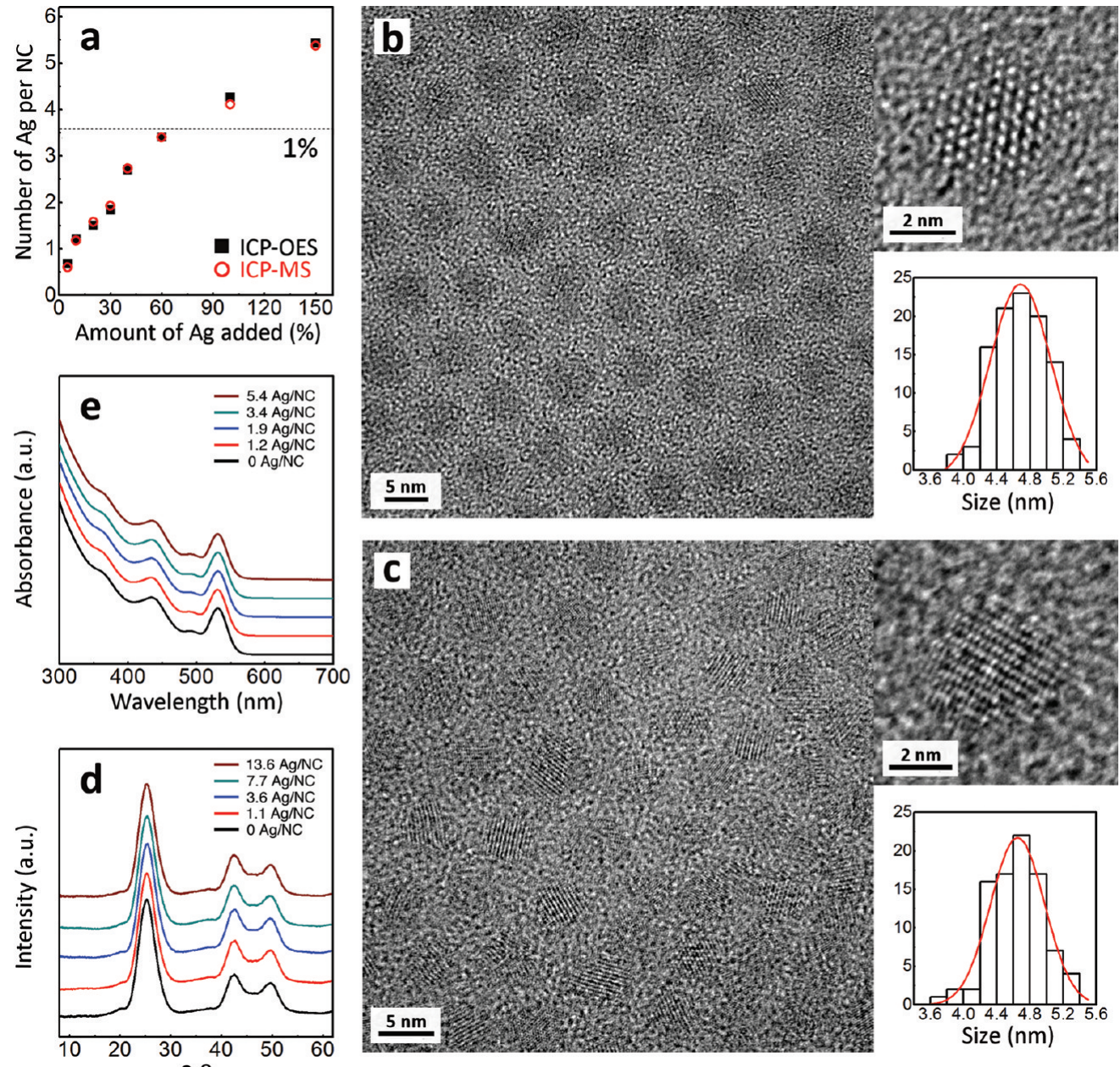

Figure 1. (a) The number of $\mathrm{Ag}$ atoms incorporated per 2.7-nm-diameter CdSe nanocrystal (NC), determined via ICP-OES and ICP-MS, versus the amount of $\mathrm{Ag}$ added to the exchange solution, as a percent of the total Cd present. (b) Electron micrograph of undoped 4.7-nm-diameter CdSe nanocrystals. (c) Electron micrograph of the same nanocrystals as in part b but doped with $\sim 12 \mathrm{Ag} / \mathrm{NC}(0.60 \% \mathrm{Ag})$. The insets in parts $\mathrm{b}$ and $\mathrm{c}$ show single-crystalline particles with size histograms that are unaltered by doping. (d) X-ray diffraction patterns for 3.0-nm-diameter CdSe nanocrystals with no $\mathrm{Ag}$ (black), $1.1 \mathrm{Ag} / \mathrm{NC}(0.20 \% \mathrm{Ag}$, red), $3.6 \mathrm{Ag} / \mathrm{NC}(0.67 \% \mathrm{Ag}$, blue), $7.7 \mathrm{Ag} / \mathrm{NC}(1.4 \% \mathrm{Ag}, \mathrm{green})$, and $13.6 \mathrm{Ag} / \mathrm{NC}(2.5 \%$ $\mathrm{Ag}$, brown). (e) Room-temperature absorption spectra of 2.7-nm-diameter CdSe nanocrystals dispersed in hexanes with no $\mathrm{Ag}$ (black), $1.2 \mathrm{Ag} / \mathrm{NC}$ (0.32\% Ag, red), $1.9 \mathrm{Ag} / \mathrm{NC}(0.53 \% \mathrm{Ag}$, blue), $3.4 \mathrm{Ag} / \mathrm{NC}$ (0.93\% Ag, green), and $5.4 \mathrm{Ag} / \mathrm{NC}$ (1.5\% Ag, brown).

small volume provides an additional motivation to study their physical properties.

As shown below, the results on our samples not only confirm electronic impurity doping in $\mathrm{CdSe}$, but they also reveal unexpected complexity. The addition of even one impurity per nanocrystal causes a dramatic enhancement in the fluorescence efficiency. At slightly higher concentrations, a dopant-related fluorescence peak appears that dominates at cryogenic temperatures. In electrical measurements on films of $\mathrm{Ag}$ doped CdSe nanocrystals, we observe nonmonotonic shifts in the Fermi energy as a function of dopant concentration. We argue that all of these findings can be explained by an unexpected transition from $\mathrm{n}$ - to p-type doping for $\mathrm{Ag}$ with increasing impurity concentration.

A priori, one would expect $\mathrm{Ag}^{+}$to be a substitutional impurity, replacing $\mathrm{Cd}^{2+}$ in the CdSe lattice. In that case, the dopant could provide an extra hole due to its deficiency in valence electrons. In other words, Ag should be an acceptor (ptype impurity). Indeed, in the previous report on Ag-doped InAs nanocrystals, all of the observed trends in the high-doping limit were rationalized by p-type doping due to the substitution of $\mathrm{Ag}^{+}$for $\mathrm{In}^{3+}$ in the lattice. ${ }^{19}$ Of course, as discussed further below, bulk studies have shown that impurity centers in semiconductors can be quite complex and it is difficult to predict whether Ag will be p- or n-type in either InAs or CdSe nanocrystals. Thus, experiments must be performed, particularly in nanocrystals where quantum confinement and surface effects can lead to new physics for the dopant.

To begin, we prepared lightly doped nanocrystals by adapting a standard cation-exchange procedure for nanocrystals. $^{28-30}$ Previously, it was shown that CdSe nanocrystals that were exposed to $\mathrm{Ag}$ cations in solution could be completely or partially converted to $\mathrm{Ag}_{2} \mathrm{Se}$. However, due to the high efficiency of this exchange, it proved difficult to apply to doping at lower concentrations because the nanocrystals can quickly become heavily doped or even multiphase. To avoid this, we included trioctylphosphine as a surfactant during the exchange to mediate the incorporation. Ethanolic $\mathrm{AgNO}_{3}$ was mixed with trioctylphosphine and added to a dispersion of preprepared CdSe nanocrystals ${ }^{31}$ with mild heating. The resulting nanocrystals were then isolated and dispersed in toluene. Nanocrystals with less than $\sim 20 \mathrm{Ag}$ per nanocrystal 

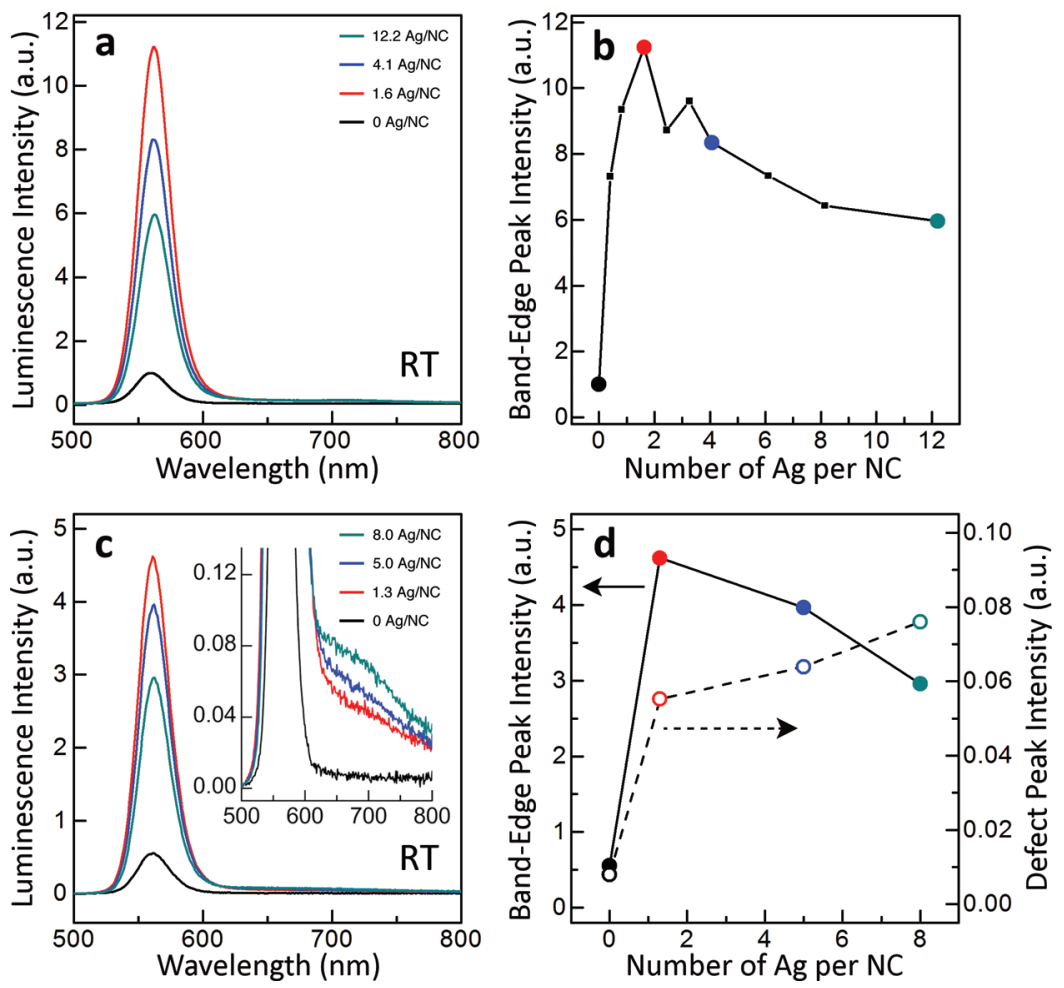

Figure 2. (a) Room-temperature (RT) fluorescence spectra of 3.1-nm-diameter CdSe nanocrystals (NCs) dispersed in hexanes with no Ag (black), $1.6 \mathrm{Ag} / \mathrm{NC}(0.3 \% \mathrm{Ag}$, red), $4.1 \mathrm{Ag} / \mathrm{NC}(0.74 \% \mathrm{Ag}$, blue), and $12.2 \mathrm{Ag} / \mathrm{NC}(2.22 \% \mathrm{Ag}$, green). The excitation wavelength was $350 \mathrm{~nm}$. (b) The intensity of the band-edge fluorescence peak near $560 \mathrm{~nm}$ plotted as a function of the number of $\mathrm{Ag}$ per nanocrystal. The colored data points correspond to the spectra in part a. (c) RT data for 3.0-nm-diameter CdSe nanocrystals dispersed in hexanes, plotted as in part a, with no Ag (black), $1.3 \mathrm{Ag} / \mathrm{NC}(0.26 \% \mathrm{Ag}$, red), $5.0 \mathrm{Ag} / \mathrm{NC}(1.0 \% \mathrm{Ag}$, blue), and $8.0 \mathrm{Ag} / \mathrm{NC}(1.5 \% \mathrm{Ag}$, green). The inset magnifies the weak fluorescence feature near $700 \mathrm{~nm}$. (d) The intensity of the band-edge fluorescence peak near $560 \mathrm{~nm}$ (solid line) and the defect-related peak near $700 \mathrm{~nm}$ (dashed line), plotted as in part $\mathrm{b}$. The colored data points correspond to the spectra in part $\mathrm{c}$.

$(<4 \% \mathrm{Ag}$ to $\mathrm{Cd})$ were stable for months under ambient. At higher concentrations, Ag films appeared on glass storage vials after several weeks, which has also been seen in $\mathrm{Ag}_{2} \mathrm{Se}$ nanocrystals. ${ }^{32}$

One key advantage of the cation-exchange approach is that it automatically provides a control sample for determining changes due to doping. In other syntheses, where the impurities are added during the nanocrystal growth, it is challenging to prepare an undoped sample of the same size, shape, and quality. Furthermore, with cation exchange, an entire series of samples that only differ by the amount of Ag that is incorporated can be easily prepared. In Figure 1a, we plot the average number of $\mathrm{Ag}$ in 2.7-nm-diameter CdSe nanocrystals versus the amount of $\mathrm{AgNO}_{3}$ in the exchange solution. To quantify Ag incorporation, we used both inductively coupled plasma optical emission spectroscopy (ICP-OES) and mass spectroscopy (ICP-MS). We observed a consistent monotonic increase in the incorporated dopant with increasing $\mathrm{AgNO}_{3}$. Thus, dopant concentration could be easily controlled in the lightly doped limit.

Figure $1 b, c$ shows transmission electron micrographs of 4.7$\mathrm{nm}$-diameter CdSe nanocrystals before and after the exchange process. This doped sample has a large number $(\sim 12)$ of $\mathrm{Ag}$ per nanocrystal. This was chosen to confirm that the particles are still highly crystalline (with no noticeable increase in stacking faults or twins) and have a size distribution that is unaltered even at this high concentration. X-ray diffraction patterns (Figure 1d) and room-temperature absorption spectra
(Figure 1e) also show no significant changes as a function of dopant concentration.

However, dramatic changes were observed when the nanocrystal fluorescence was analyzed. Figure 2 a shows room-temperature emission spectra for 3.1-nm-diameter CdSe nanocrystals dispersed in hexanes as a function of the average number of Ag per nanocrystal. To compare the data, we first matched the absorbance for all samples at the lowest-energy electronic transition by controlling the nanocrystal concentration in the dispersion. The addition of the $\mathrm{Ag}$ clearly enhances the fluorescence, with the maximum intensity (Figure $2 \mathrm{~b})$ peaking around $2 \mathrm{Ag}$ per nanocrystal. Additional $\mathrm{Ag}$ then decreases the intensity from this maximum. This same trend, an enhancement of the fluorescence with the addition of a few $\mathrm{Ag}$ per nanocrystal and then a slow decrease at higher concentrations, was observed consistently across different samples and sizes (Figure 2d and Figures $\mathrm{S} 1-\mathrm{S} 8$ in the Supporting Information). The magnitude of the initial increase depended on how much the nanocrystals had been cleaned, but we verified that the addition of trioctylphosphine alone (i.e., the replenishment of the surface ligand) could not explain the increase (Figure S8, Supporting Information). Furthermore, we quantified the fluorescence quantum yield for the 3.5-nmdiameter sample shown in Figure S6 (Supporting Information) and observed a jump from 14 to $27 \%$ with the addition of 1.2 $\mathrm{Ag}$ per nanocrystal. Note that the quantum yield of CdSe nanocrystals with $\sim 2 \mathrm{Ag}$ atoms is comparable to that of $\mathrm{CdSe} /$ $\mathrm{ZnS}$ core/shell nanocrystals. ${ }^{33,34}$ 

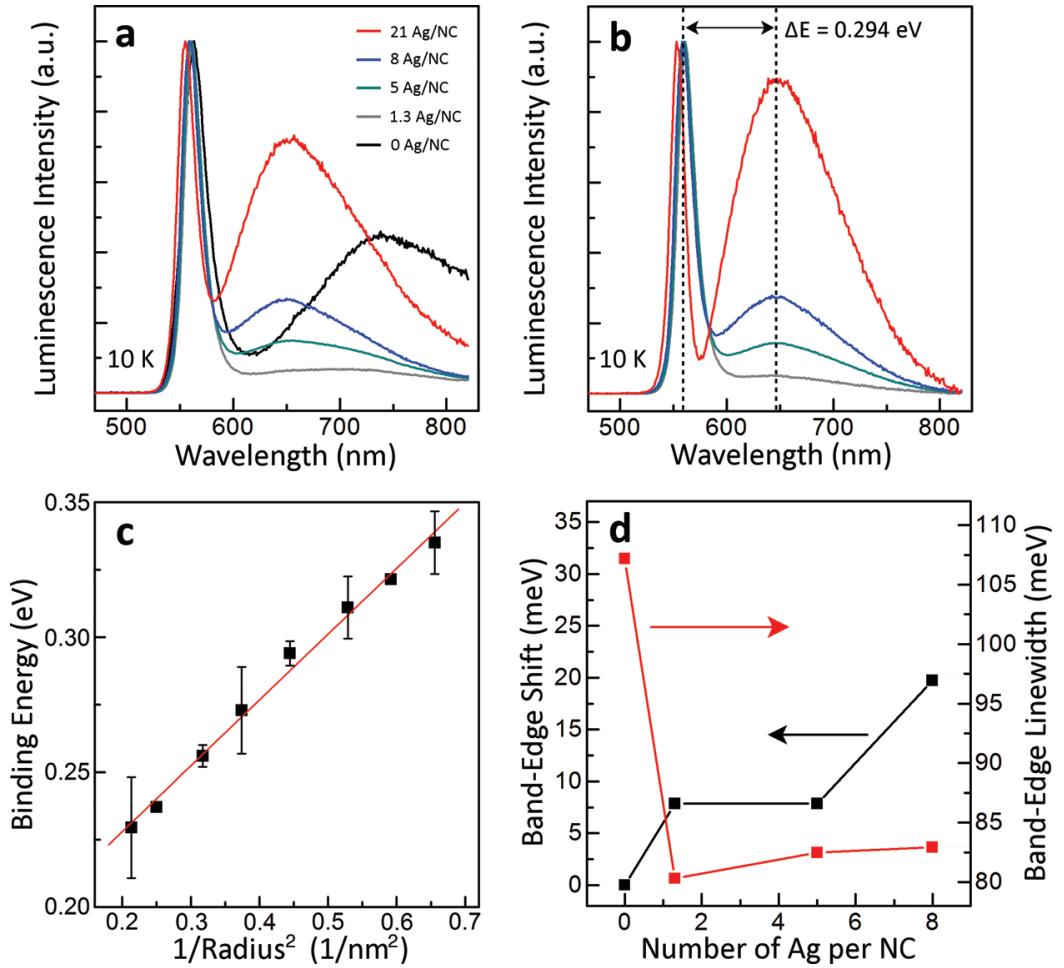

Figure 3. (a) Fluorescence spectra of the 3.0-nm-diameter CdSe nanocrystals (NCs) from Figure $2 \mathrm{c}$ cast as neat films and cooled to $10 \mathrm{~K}$. The samples have no $\mathrm{Ag}$ (black), $1.3 \mathrm{Ag} / \mathrm{NC}(0.26 \% \mathrm{Ag}$, gray), $5.0 \mathrm{Ag} / \mathrm{NC}(1.0 \% \mathrm{Ag}$, green), $8.0 \mathrm{Ag} / \mathrm{NC}$ (1.5\% Ag, blue), and $21 \mathrm{Ag} / \mathrm{NC}(4.0 \% \mathrm{Ag}, \mathrm{red})$. The spectra were normalized to the peak of the band-edge feature. The excitation wavelength was $430 \mathrm{~nm}$. (b) Normalized spectra from part a after subtracting the deep-trap fluorescence that is present in all CdSe nanocrystals at low temperatures (see black curve in part a). The energy separation between the band-edge peak and the defect-related feature is marked with vertical lines. (c) This energy separation, which is assigned to the defect binding energy, versus the inverse nanocrystal radius squared. The values were extracted from data as in part $b$ for a range of nanocrystal sizes. A linear regression fit (red line) yields a coefficient of determination of 0.992. The error bars represent the standard deviation from averaging two to five samples for each size. Data points without error bars are for one sample. (d) The extracted line width (red) and energy shift (black) of the bandedge fluorescence peak versus the number of $\mathrm{Ag}$ per nanocrystal.

The rise in the fluorescence intensity with the addition of a few dopants is quite unexpected. For example, it was recently reported that the fluorescence of $\mathrm{CdSe} / \mathrm{CdS}$ quantum dots/ rods is quenched 100-fold with the introduction of $\mathrm{Cu}$ impurities. $^{35}$ To explain our enhancement, several possible mechanisms can be considered. First, each Ag atom could provide an extra carrier (either electron or hole) to one of the quantum-confined levels of the nanocrystal and enhance the radiative rate. This would be surprising because the presence of the extra carrier should cause rapid nonradiative Auger recombination, ${ }^{36}$ which should decrease the fluorescence intensity. Nevertheless, an increase in fluorescence intensity has been observed in CdSe nanocrystals in which an extra electron was injected via electrochemical doping. ${ }^{8}$ In that case, the increase was explained by the presence of a negatively charged exciton (or negative trion, $\mathrm{X}^{-}$), which should have a faster radiative rate than a neutral exciton. Further, it has recently been argued that Auger recombination is much less efficient for the negative trion compared to the positive trion, ${ }^{37}$ which in principle could rationalize why the fluorescence is not quenched with the addition of an extra carrier. This picture would then suggest that the Ag acts as a donor and provides an extra electron. However, this explanation suffers from several inconsistencies with the data. The presence of an extra electron in a quantum-confined level would induce a bleach in the lowest energy absorption feature, ${ }^{6}$ which we do not observe (Figure 1e). An increase of the fluorescence by a factor of $\sim 10$ is also difficult to explain quantitatively based on the negative trion model. ${ }^{38}$

A second explanation is that the $\mathrm{Ag}$ atoms passivate nonradiative traps, either by binding to the nanocrystal surface directly or by incorporating into the nanocrystal and providing extra carriers that then fill these traps. However, the data would then suggest that in all samples only $\sim 2$ traps must be filled to have a dramatic effect. While possible, we believe this explanation is unlikely.

Rather, we propose that a third mechanism is more plausible. We must recognize that a fundamental difference exists between impurity doping and electrochemical doping. In impurity doping, one does not just add an extra carrier but also the impurity center as well. If the extra carrier fills a trap (or otherwise escapes from the nanocrystal, for example, by reducing/oxidizing some chemical species on the nanocrystal surface or in the surrounding matrix ${ }^{25}$ ), this will leave behind an ionized impurity center in the nanocrystal. Even at one or two impurities, the electrostatic field of such centers can presumably influence the fluorescence. For example, positively charged CdSe nanocrystals have recently been invoked to explain nanocrystals with unusual brightness. ${ }^{39,40}$ If the $\mathrm{Ag}$ atoms provide extra electrons, which then leave to traps outside the nanocrystals, this would induce a positively charged impurity center, which could enhance the brightness. This would suggest that $\mathrm{Ag}$ is acting as an n-type donor.

In addition to the initial rise, the fluorescence spectra also reveal a weak feature on the long-wavelength side of the main 

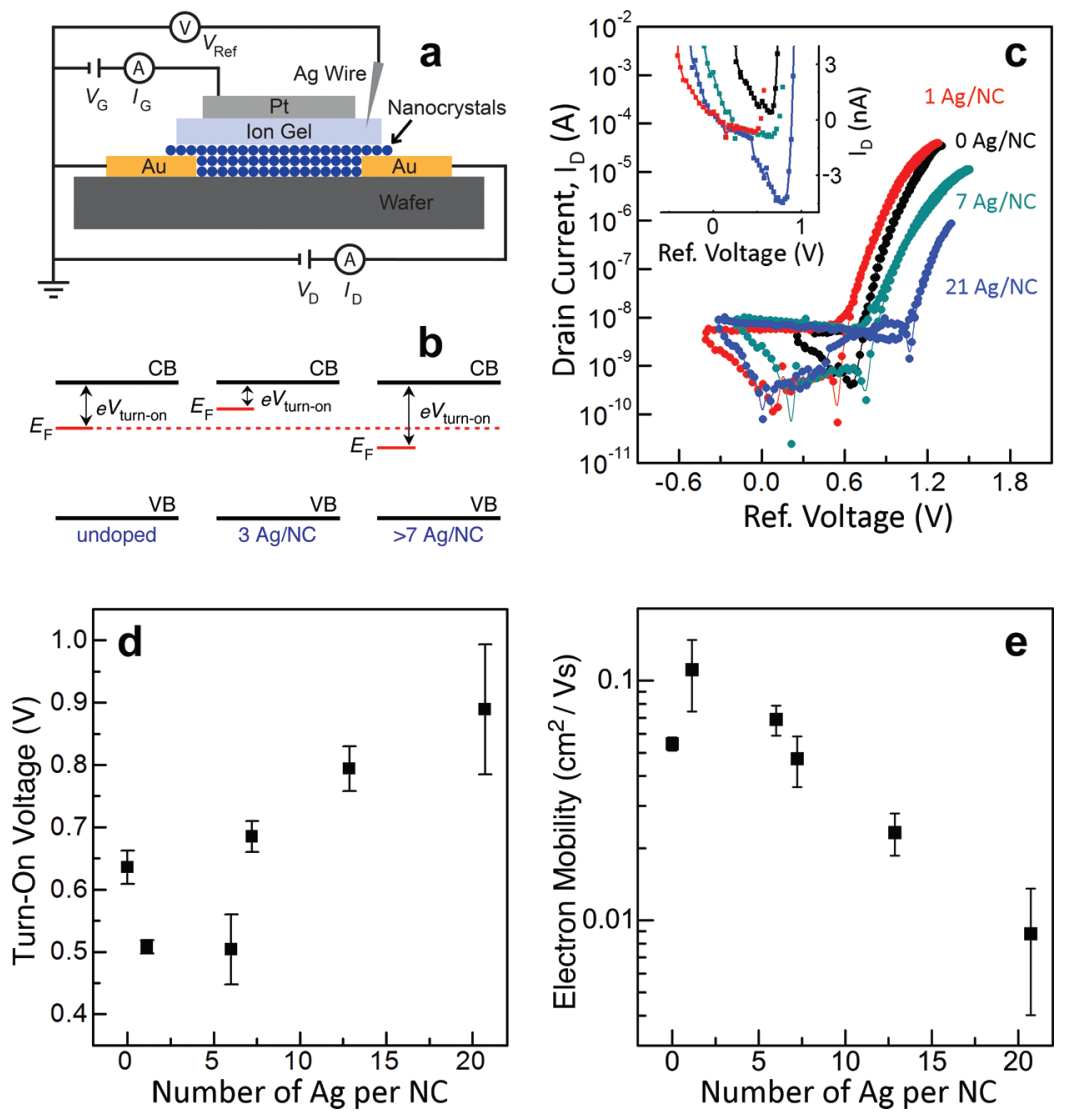

Figure 4. (a) Schematic cross section (not to scale) of the ion-gel-gated thin-film transistors used to characterize the electrical properties of the doped nanocrystals (NCs). The length and width of the channel were $10 \mu \mathrm{m}$ and $1 \mathrm{~mm}$, respectively. (b) Energy-level diagram depicting the relationship between the Fermi energy, the turn-on voltage, and the conduction band for films of CdSe nanocrystals (undoped, $\sim 3 \mathrm{Ag} / \mathrm{NC}$, and $>7$ $\mathrm{Ag} / \mathrm{NC}$ ). (c) Absolute value of the drain current, $I_{\mathrm{D}}$, versus the reference voltage, $V_{\text {ref }}$, for 3.6-nm-diameter nanocrystals with no Ag (black), $1.0 \mathrm{Ag} /$ $\mathrm{NC}\left(0.13 \% \mathrm{Ag}\right.$, red), $7.0 \mathrm{Ag} / \mathrm{NC}\left(0.85 \% \mathrm{Ag}\right.$, green), and $21 \mathrm{Ag} / \mathrm{NC}(2.4 \% \mathrm{Ag}$, blue $) . V_{\mathrm{D}}$ was $0.1 \mathrm{~V}$, and $V_{\text {ref }}$ was measured from an oxidized silver wire in the ion gel. The inset shows a magnified plot of the drain current near the turn-on voltage. (d) The turn-on voltage versus the number of Ag per nanocrystal, extracted from data as in part c. (e) The electron mobility computed at a gate voltage of $2.5 \mathrm{~V}\left(\mathrm{a}\right.$ carrier concentration of $\left.10^{14} / \mathrm{cm}^{2}\right)$ versus the number of $\mathrm{Ag}$ per nanocrystal, extracted from data as in part $\mathrm{c}$. The error bars in parts $\mathrm{d}$ and e represent the standard deviation from averaging two to five devices. Data points without error bars are for one device.

"band-edge" emission peak (Figure 2c). This weak feature appears only in the doped samples, increases in intensity with dopant concentration (Figure $2 \mathrm{~d}$ ), and was present in all sizes of Ag-doped CdSe nanocrystals (Figures S3-S8 in the Supporting Information). However, because its intensity is 30-100 times less than the main peak, an analysis is challenging from room-temperature data. Therefore, we cast neat films of the nanocrystals on sapphire discs and acquired fluorescence spectra at $10 \mathrm{~K}$. The results, shown in Figure 3a, are normalized to the intensity of the band-edge peak. The weak feature is more intense at low temperature and becomes prominent at higher doping. To resolve this feature further, we subtracted the "deep-trap" fluorescence that is present in all CdSe nanocrystals at $10 \mathrm{~K}$ (see the black curve for the undoped sample in Figure 3a). The resulting spectra (Figure 3b) show a dopant-related peak separated from the band-edge emission by a constant $294 \mathrm{meV}$.

One possible origin for this dopant-related peak is the recombination of carriers that are bound to the impurity with those that are optically excited into the lowest quantumconfined level. The separation would then be related to the binding energy of the impurity. To explore this, we measured the low-temperature fluorescence spectra for different sizes of Ag-doped CdSe nanocrystals and extracted the separation between the dopant-related peak and the band-edge emission. If the separation is due to the binding energy, its size dependence should depend on the confinement regime of the dopant. ${ }^{41}$ If the dopant is weakly confined, its binding energy should scale as $1 / R^{2}$, where $R$ is the nanocrystal radius. If the dopant is strongly confined, it should go as $1 / R$. Assuming hydrogenic impurities, an acceptor in CdSe would have a Bohr radius of $\sim 0.7 \mathrm{~nm}$. Thus, in our size regime, it should be weakly confined and yield a $1 / R^{2}$ size dependence. In contrast, a donor in CdSe would have a Bohr radius of $\sim 5.0 \mathrm{~nm}$, would be strongly confined, and would lead to a $1 / R$ dependence. Figure $3 c$ and Figure $S 9$ in the Supporting Information plot the data versus $1 / R^{2}$ and $1 / R$, respectively. Unfortunately, the data fit well to either dependence (with coefficients of determination of 0.992 and 0.997 ). Consequently, it is difficult to distinguish the two cases from the size dependence. However, if we extrapolate each fit to infinite size, we can estimate what each scaling would predict for the binding energy of $\mathrm{Ag}$ in bulk CdSe. We extract values of 180 and $83 \mathrm{meV}$, respectively. These values are much closer to what is expected ${ }^{42,43}$ for a hydrogenic acceptor ( 84 $\mathrm{meV})$ than for a hydrogenic donor $(13 \mathrm{meV})$ in CdSe. Thus, keeping in mind all of the assumptions in the above analysis, this defect-related peak appears to be more consistent with $\mathrm{Ag}$ as an acceptor. The dopant-related peak would then be 
explained by the recombination of photoexcited electrons with Ag-bound holes. In this case, at higher concentrations, where the dopant-related peak becomes significant, $\mathrm{Ag}$ is acting as a ptype dopant.

At this point, it may be helpful to summarize our interpretation of the optical data. At low doping of $\mathrm{Ag}$, we argue that the impurity is an n-type dopant. Because the donor electrons are quickly trapped outside the nanocrystals, the particles become positively charged due to the ionized impurity. Without extra electrons in the conduction band states of the nanocrystals, one would not expect changes in the absorption spectra (Figure 1e) or Auger quenching. ${ }^{36}$ At the same time, the positively charged impurity center can explain the enhanced photoluminescence. At higher doping, we argue that the $\mathrm{Ag}$ begins to behave as a p-type dopant. Because of the strong binding energy and localized character of a hydrogenic acceptor in $\mathrm{CdSe}$, a small population of the extra holes remain bound to the impurity center even at room temperature. This gives rise to the weak dopant-related shoulder in Figure $2 \mathrm{c}$ when photoexcited electrons recombine with these holes. At $10 \mathrm{~K}$, thermal activation is diminished, and a much larger percent of the holes remain bound, leading to the more pronounced dopant-related peak in Figure $3 b$.

Before discussing this further, we note that other subtle changes were observed in the fluorescence with doping (Figure 3d). At $10 \mathrm{~K}$, the band-edge peak shifts to higher energy; its line width decreases by $\sim 25 \%$ with the incorporation of just 1.3 $\mathrm{Ag}$ per nanocrystal $(0.26 \% \mathrm{Ag})$. While the exact origin of these effects is not yet clear, both the blue shift and line narrowing in our lightly doped samples are in contrast to observations in the heavily doped InAs nanocrystals. ${ }^{19}$ There, room-temperature red shifts and peak broadening were explained by band tailing due to high doping. At room temperature, our samples exhibit essentially no change in peak position or line width in the lowdoping limit (<10 Ag per nanocrystal).

While the optical results clearly show that the addition of a few Ag per nanocrystal can cause large changes, our interpretation, in which $\mathrm{Ag}$ acts as an n-type dopant (donor) at very low concentrations and then transitions to a p-type donor (acceptor) as the number of Ag per nanocrystal is increased, is fairly speculative. If such a doping trend occurs, however, it should also affect electrical transport. Thus, we fabricated electrolyte-gated thin-film transistors to probe the conductivity of films of Ag-doped CdSe nanocrystals. Figure 4a displays a schematic cross section of the device. ${ }^{44}$ Films of nanocrystals $\left(\sim 50 \mathrm{~nm}\right.$ thick) were spin-coated onto $\mathrm{Si} / \mathrm{SiO}_{2}$ substrates that were prepatterned with source and drain electrodes $(\mathrm{Cr} / \mathrm{Au})$. Since the as-deposited films are insulating due to the bulky ligands on the nanocrystal surfaces, we first treated the films with methanolic sodium hydroxide to remove the ligands and improve conduction. ${ }^{45,46}$ The resulting films are n-type even without $\mathrm{Ag}$ doping. Before testing the influence of $\mathrm{Ag}$, we first verified that the $\mathrm{NaOH}$ treatment did not affect the number of Ag per nanocrystal in the films (Figure S10 in the Supporting Information). For the gate dielectric, we employed an ion gel composed of $10 \mathrm{wt} \%$ triblock copolymer poly(styrene-block-methylmethacrylate-block-styrene) and 90 wt \% ionic liquid 1-ethyl-3-methylimidazolium bis(trifluoromethylsulfonyl)imide. ${ }^{47}$ The gel was spread over the channel region of the device. On top of the ion-gel layer, a Pt foil was attached as the gate electrode and electrical measurements were performed in an evacuated probe station $\left(\sim 10^{-6}\right.$ Torr $)$.
Our devices also included an oxidized silver wire as a reference electrode. This can measure the potential drop across the interface between the ion gel and the nanocrystal film under gate bias. ${ }^{44}$ Thus, the local potential in each film at which conduction turned on could be accurately determined. Because this turn-on voltage is directly related to the energy offset between the Fermi level in the film and the conducting states in the nanocrystals at zero bias (Figure 4b), it can give very useful information about the influence of dopants. For example, Figure $4 \mathrm{c}$ shows measurements for transistors made from 3.6nm-diameter CdSe nanocrystals at five different dopant concentrations. The drain current $\left(I_{\mathrm{D}}\right)$ is plotted as a function of the voltage at the reference electrode $\left(V_{\text {ref }}\right)$. As in previous studies, our film of undoped CdSe nanocrystals started to conduct when the interface potential $\left(V_{\text {ref }}\right)$ was biased positively. More specifically, $I_{D}$ increased sharply with increasing positive voltage, which indicates that the charge carriers in these films are negatively charged (i.e. electrons). More importantly, for doped films, Figure $4 \mathrm{c}$ shows that the turn-on voltage varies with impurity concentration (see inset). Assuming that the energy of the lowest electronic level $\left(1 S_{\mathrm{e}}\right)$ of the nanocrystals does not change significantly with light doping, the shift in the turn-on voltage for different doping concentrations is then a direct observation of changes in the Fermi level (Figure $4 \mathrm{~b}$ ).

Figure $4 \mathrm{~d}$ plots the variation in the turn-on voltage for the same 3.6-nm-diameter CdSe nanocrystals at six Ag concentrations. At low doping, we observe that the turn-on voltage is smaller than that of the undoped CdSe nanocrystals. This indicates that the Fermi level shifts closer to the lowest electronic level of the nanocrystals (Figure $4 \mathrm{~b}$ ), which is a signature of $\mathrm{n}$-type doping. (We have also recently reported a similar shift in Al-doped CdSe nanocrystals, ${ }^{24}$ where $\mathrm{Al}$ is expected to be an n-type dopant.) For Ag, we see this behavior below $\sim 6 \mathrm{Ag}$ per nanocrystal. At higher dopant concentrations, the turn-on voltage reverses direction and increases above the value for undoped nanocrystals. The Fermi level now shifts away from the lowest electronic level of the nanocrystals (Figure $4 \mathrm{~b}$ ), which is a signature of p-type doping. Therefore, these nonmonotonic shifts in the Fermi level support the conclusions from the optical experiments. However, we note that, while the doping clearly influenced the Fermi level, it was not possible to observe any hole conduction even at our highest dopant concentrations. It is unknown whether this is due to the inherent instability of extra holes in $\mathrm{CdSe}^{25}$ or the large excess of electrons induced by the $\mathrm{NaOH}$ treatment. Unfortunately, our transistors were not gateable without the $\mathrm{NaOH}$ treatment.

In addition to the possibility of Fermi-level shifts, it is important to consider an alternative origin for the shifts in the turn-on voltage. Namely, it is possible that $\mathrm{Ag}$ cations are decorating the nanocrystal surface and inducing positive local electrostatic fields. The positive external voltage that is required to turn on conduction would then be diminished, as observed in Figure $4 \mathrm{~d}$ at low doping. In this case, the measured voltage shifts would have nothing to do with the introduction of extra carriers by the dopants. However, this model has several inconsistencies with the data. First, it is difficult to explain why the turn-on voltage reverses direction at higher dopant concentration with only a surface effect. Second, we observe enhanced conduction even without the gate. As shown in Figure S11 in the Supporting Information, the conductivity of films of doped CdSe nanocrystals increased by $\sim 2$ orders of magnitude with the addition of $\sim 5 \mathrm{Ag} / \mathrm{NC}$. These conductivity 
measurements were performed on films that were not exposed to $\mathrm{NaOH}$, ruling out any unexpected influence of this treatment. We also confirmed by steady-state current measurements that the enhanced conduction was not due to ionic transport of Ag cations (which would be inherently limited). Rather, the data support the conclusion that the dopants are electronically active. Indeed, we observed this enhanced conductivity early in this project. However, because these measurements are quite hysteretic (see Figure S11, Supporting Information), a detailed analysis of this effect is challenging. We therefore moved to the transistors, which allow a precise determination of the Fermi level.

Further support for our interpretation can be found in the influence of the dopant concentration on the electron mobility of the film (Figure 4e). Mobility $(\mu)$ was estimated from the equation $\mu=(L / W) \times\left(I_{\mathrm{D}} / e n V_{\mathrm{D}}\right)$, where $L$ and $W$ are the channel length and width, respectively, $e$ is the elemental charge, and $n$ is the electron concentration, determined as previously described. ${ }^{44}$ Because electron mobility also varies with carrier concentration, we fixed the gate-induced carrier density (at $10^{14}$ carriers $/ \mathrm{cm}^{2}$ ) and then compared electron mobilities for films of CdSe nanocrystals with different dopant concentrations. We observed that the electron mobility first increased at low dopant concentrations and then decreased gradually with further doping, again consistent with a transition from $\mathrm{n}$ - to p-type doping.

In bulk II-VI semiconductors, a silver atom can behave either as a p-type dopant ${ }^{48-54}$ when it substitutes for the cation or as an n-type dopant ${ }^{49,50,55,56}$ when it occupies an interstitial site. In the latter case, the valence electron from the interstitial $\mathrm{Ag}$ atom can be directly donated to the lattice. Interestingly, $\mathrm{Au}$ atoms can even form interstitial donors at low doping concentrations and substitutional acceptors at higher doping concentrations. ${ }^{57} \mathrm{~A}$ similar mechanism might be occurring for $\mathrm{Ag}$ in CdSe nanocrystals. At low doping concentrations, $\mathrm{Ag}$ atoms could act as interstitial donors. At higher concentrations, these donors could be compensated by donor-acceptor complexes, and then act as substitutional acceptors at even higher concentrations.

This mechanism could be confirmed if the local structure around the Ag dopants could be determined. In general, this is a difficult task in nanocrystals. Indeed, $\mathrm{Mn}$ impurities have been heavily studied, at least in part, because their local environment can be measured with electron paramagnetic resonance (EPR) ${ }^{58}$ For other dopants, alternative techniques must be applied. ${ }^{59}$ To address this in our system, we have begun experiments on extended X-ray absorption fine structure (EXAFS) spectroscopy to locate the $\mathrm{Ag}$ atoms within the $\mathrm{CdSe}$ lattice. Due to the low concentration of $\mathrm{Ag}$, the experiments are extremely challenging. So far, our preliminary analyses for data from samples with $3.6 \mathrm{Ag}$ per nanocrystal suggest only that the spectra cannot be fit with the following: (i) Ag placed in only substitutional sites of the CdSe lattice or (ii) $\mathrm{Ag}$ placed in a separate tetragonal phase of $\mathrm{Ag}_{2} \mathrm{Se}$. The latter is observed if the cation exchange process leads to multiphase $\mathrm{Ag}_{2} \mathrm{Se}-\mathrm{CdSe}$ nanocrystals. ${ }^{32}$ In other words, the data suggests that, at low concentrations, the $\mathrm{Ag}$ atoms are not substitutional dopants only and they do not form a separate $\mathrm{Ag}_{2} \mathrm{Se}$ phase. Therefore, these preliminary findings are so far consistent with our model of having both interstitial and substitutional placement of Ag. However, further analyses and EXAFS experiments are required to determine the exact local structure of the Ag.
In combination, our optical and electrical data indicate that $\mathrm{Ag}$ is an electronically active impurity in CdSe nanocrystals. More generally, they suggest that, if dopants are added to nanocrystals, the creation of $\mathrm{n}$ - and p-type films of semiconductor nanocrystals should be attainable. Our specific observations also lead to several surprising conclusions about doped nanocrystals in the lightly doped limit. First, even the first few dopants can provide electrons that alter the electrical properties of the films. Under what conditions these are available to fill band-edge electronic states, impurity-bound states, or traps is not yet known. Second, even within a small particle, the behavior of the dopant is very complicated and does not follow simple predictions. Perhaps this should have been expected, since the dopant-site energetics can be more complicated than in the bulk material due to the proximity of the nanocrystal surface. ${ }^{60}$ Certainly, these results show that further theoretical and experimental work is needed to understand specific dopant-semiconductor pairs in nanocrystals, both for fundamental understanding and use in applications.

\section{ASSOCIATED CONTENT}

\section{S Supporting Information}

Experimental methods and additional figures that support the characterization of the reported materials. This material is available free of charge via the Internet at http://pubs.acs.org.

\section{AUTHOR INFORMATION}

\section{Corresponding Author}

*E-mail: dnorris@ethz.ch.

\section{Notes}

The authors declare no competing financial interest.

\section{ACKNOWLEDGMENTS}

The authors wish to thank Al. Efros, S. Erwin, S. Crooker, D. Talapin, V. Klimov, and F. Ott for stimulating discussions and R. Knurr for assistance with the elemental analysis. This work was supported by the Materials World Network of the US National Science Foundation (NSF) and the German Research Foundation (DFG) under Award Nos. DMR-0908629 and WI 981/11. Additional support was provided by the MRSEC Program of the NSF under DMR-0819885. A.S. and M.S.K. received further funding from the Industrial Partnership for Research in Interfacial and Materials Research at the University of Minnesota (UMN) and a Doctoral Dissertation Fellowship from UMN, respectively. We utilized resources at the UMN Characterization Facility and the UMN Nanofabrication Center, which receive partial support from the NSF under the NNIN program. Preliminary EXAFS measurements were performed at the HASYLAB at DESY in Hamburg, Germany. The authors thank the staff of the X1 beamline, particularly A. Webb and M. Murphy, for their support.

\section{REFERENCES}

(1) Alivisatos, A. P. Science 1996, 271, 933.

(2) Klimov, V. I. Nanocrystal Quantum Dots, 2nd ed.; CRC Press: Boca Raton, FL, 2010.

(3) Gur, I.; Fromer, N. A.; Geier, M. L.; Alivisatos, A. P. Science 2005, $310,462$.

(4) Coe, S.; Woo, W. K.; Bawendi, M.; Bulovic, V. Nature 2002, 420, 800.

(5) Shim, M.; Guyot-Sionnest, P. Nature 2000, 407, 981.

(6) Yu, D.; Wang, C. J.; Guyot-Sionnest, P. Science 2003, 300, 1277.

(7) Talapin, D. V.; Murray, C. B. Science 2005, 310, 86. 
(8) Wang, C.; Shim, M.; Guyot-Sionnest, P. Science 2001, 291, 2390.

(9) Roest, A. L.; Kelly, J. J.; Vanmaekelbergh, D.; Meulenkamp, E. A. Phys. Rev. Lett. 2002, 89, 036801.

(10) Stowell, C. A.; Wiacek, R. J.; Saunders, A. E.; Korgel, B. A. Nano Lett. 2003, 3, 1441.

(11) Orlinskii, S. B.; Schmidt, J.; Baranov, P. G.; Hofmann, D. M.; de Mello Donegá, C.; Meijerink, A. Phys. Rev. Lett. 2004, 92, 047603.

(12) Meulenberg, R. W.; van Buuren, T.; Hanif, K. M.; Willey, T. M.; Strouse, G. F.; Terminello, L. J. Nano Lett. 2004, 4, 2277.

(13) Fujii, M.; Yamaguchi, Y.; Takase, Y.; Ninomiya, K.; Hayashi, S. Appl. Phys. Lett. 2005, 87, 211919.

(14) Stegner, A. R.; Pereira, R. N.; Klein, K.; Lechner, R.; Dietmueller, R.; Brandt, M. S.; Stutzmann, M.; Wiggers, H. Phys. Rev. Lett. 2008, 100, 026803.

(15) Pi, X. D.; Gresback, R.; Liptak, R. W.; Campbell, S. A.; Kortshagen, U. Appl. Phys. Lett. 2008, 92, 123102.

(16) Roy, S.; Tuinenga, C.; Fungura, F.; Dagtepe, P.; Chikan, V.; Jasinski, J. J. Phys. Chem. C 2009, 113, 13008.

(17) Geyer, S. M.; Allen, P. M.; Chang, L. Y.; Wong, C. R.; Osedach, T. P.; Zhao, N.; Bulovic, V.; Bawendi, M. G. ACS Nano 2010, 4, 7373.

(18) Ishizumi, A.; Kanemitsu, Y. J. Phys. Soc. Jpn. 2010, 79, 093706.

(19) Mocatta, D.; Cohen, G.; Schattner, J.; Millo, O.; Rabani, E.; Banin, U. Science 2011, 332, 77.

(20) Luther, J. M.; Jain, P. K.; Ewers, T.; Alivisatos, A. P. Nat. Mater. 2011, 10, 361.

(21) Srivastava, B. B.; Jana, S.; Pradhan, N. J. Am. Chem. Soc. 2011, 133, 1007.

(22) Buonsanti, R.; Llordes, A.; Aloni, S.; Helms, B. A.; Milliron, D. J. Nano Lett. 2011, 11, 4706.

(23) Viswanatha, R.; Brovelli, S.; Pandey, A.; Crooker, S. A.; Klimov, V. I. Nano Lett. 2011, 11, 4753.

(24) Wills, A. W.; Kang, M. S.; Wentz, K. M.; Hayes, S. E.; Sahu, A.; Gladfelter, W. L.; Norris, D. J. J. Mater. Chem. 2012, 22, 6335.

(25) Norris, D. J.; Efros, Al. L.; Erwin, S. C. Science 2008, 319, 1776.

(26) Beulac, R.; Ochsenbein, S. T.; Gamelin, D. R. Colloidal Transition-Metal-Doped Quantum Dots. In Nanocrystal Quantum Dots; Klimov, V. I., Ed.; CRC Press: Boca Raton, FL, 2010; p 397.

(27) Koenraad, P. M.; Flatté, M. E. Nat. Mater. 2011, 10, 91.

(28) Son, D. H.; Hughes, S. M.; Yin, Y.; Alivisatos, A. P. Science 2004, 306, 1009.

(29) Mokari, T.; Aharoni, A.; Popov, I.; Banin, U. Angew. Chem., Int. Ed. 2006, 45, 8001.

(30) Robinson, R. D.; Sadtler, B.; Demchenko, D. O.; Erdonmez, C. K.; Wang, L.-W.; Alivisatos, A. P. Science 2007, 317, 355.

(31) Reiss, P.; Bleuse, J.; Pron, A. Nano Lett. 2002, 2, 781.

(32) Sahu, A.; Qi, L.; Kang, M. S.; Deng, D.; Norris, D. J. J. Am. Chem. Soc. 2011, 133, 6509.

(33) Hines, M. A.; Guyot-Sionnest, P. J. Phys. Chem. 1996, 100, 468.

(34) Dabbousi, B. O.; Rodriguez-Viejo, J.; Mikulec, F. V.; Heine, J. R.; Mattoussi, H.; Ober, R.; Jensen, K. F.; Bawendi, M. G. J. Phys. Chem. B 1997, 101, 9463.

(35) Jain, P. K.; Beberwyck, B. J.; Fong, L.-K.; Polking, M. J.; Alivisatos, A. P. Angew. Chem. 2012, 51, 2387.

(36) Klimov, V. I.; Mikhailovsky, A. A.; McBranch, D. W.; Leatherdale, C. A.; Bawendi, M. G. Science 2000, 287, 1011.

(37) Park, Y. S.; Malko, A. V.; Vela, J.; Chen, Y.; Ghosh, Y.; GarciaSantamaria, F.; Hollingsworth, J. A.; Klimov, V. I.; Htoon, H. Phys. Rev. Lett. 2011, 106, 187401.

(38) Jha, P. P.; Guyot-Sionnest, P. ACS Nano 2009, 3, 1011.

(39) Wang, X. Y.; Ren, X. F.; Kahen, K.; Hahn, M. A.; Rajeswaran, M.; Maccagnano-Zacher, S.; Silcox, J.; Cragg, G. E.; Efros, Al. L.; Krauss, T. D. Nature 2009, 459, 686.

(40) Spinicelli, P.; Buil, S.; Quelin, X.; Mahler, B.; Dubertret, B.; Hermier, J. P. Phys. Rev. Lett. 2009, 102, 136801.

(41) Ekimov, A. I.; Kudryavtsev, I. A.; Ivanov, M. G.; Efros, Al. L. J. Lumin. 1990, 46, 83.

(42) Pankove, J. I. Optical Processes in Semiconductors; Dover: New York, 1971; p 34.
(43) Ge'lmont, B. L.; D’yakonov, M. I. Sov. Phys. Semicond. 1972, 5, 1905.

(44) Kang, M. S.; Sahu, A.; Norris, D. J.; Frisbie, C. D. Nano Lett. 2010, 10, 3727.

(45) Jarosz, M. V.; Porter, V. J.; Fisher, B. R.; Kastner, M. A.; Bawendi, M. G. Phys. Rev. B 2004, 70, 195327.

(46) Yu, D.; Wehrenberg, B. L.; Jha, P.; Ma, J.; Guyot-Sionnest, P. J. Appl. Phys. 2006, 99, 104315.

(47) Cho, J. H.; Lee, J.; Xia, Y.; Kim, B.; He, Y. Y.; Renn, M. J.; Lodge, T. P.; Frisbie, C. D. Nat. Mater. 2008, 7, 900.

(48) Avinor, M.; Meijer, G. J. Chem. Phys. 1960, 32, 1456.

(49) Robinson, A. L.; Bube, R. H. J. Appl. Phys. 1971, 42, 5280.

(50) Swaminathan, V.; Greene, L. C. J. Lumin. 1976, 14, 357.

(51) Inoue, M. J. Phys. Chem. Solids 1979, 40, 857.

(52) Chamonal, J. P.; Molva, E.; Pautrat, J. L.; Revoil, L. J. Cryst. Growth 1982, 59, 297.

(53) Georgobiani, A. N.; Aminov, U. A.; Dravin, V. A.; Lepnev, L. S.; Mullabaev, I. D.; Ursaki, V. V.; Iljukhina, Z. P. Nucl. Instrum. Methods Phys. Res., Sect. A 1999, 426, 164.

(54) Sato, F.; Kagawa, T.; Yodo, Y.; Iida, T. IEEE Nucl. Sci. Symp. Conf. Rec. 2004, 1-5, 3527.

(55) Dzhafarov, T. D.; Serin, M.; Oren, D.; Sungu, B.; Sadigov, M. S. J. Phys. D: Appl. Phys. 1999, 32, L5.

(56) Laiho, R.; Lashkul, A. V.; Lahderanta, E.; Nedeoglo, D. D.; Nedeoglo, N. D.; Shakhov, M. A. Semicond. Sci. Technol. 2006, 21, 654.

(57) Nedeoglo, N. D.; Sirkeli, V. P.; Nedeoglo, D. D.; Laiho, R.; Lahderanta, E. J. Phys.: Condens. Matter 2006, 18, 8113.

(58) Kennedy, T. A.; Glaser, E. R.; Klein, P. B.; Bhargava, R. N. Phys. Rev. B 1995, 52, R14356.

(59) Gunawan, A. A.; Mkhoyan, K. A.; Wills, A. W.; Thomas, M. G.; Norris, D. J. Nano Lett. 2011, 11, 5553.

(60) Erwin, S. C.; Petukhov, A. G. Phys. Rev. Lett. 2002, 89, 227201. 